\title{
High level of ciprofloxacin resistance and its molecular background among Campylobacter jejuni strains isolated in the United Arab Emirates
}

\section{Correspondence \\ Tibor Pál \\ Tibor.Pal@aok.pte.hu}

Received 29 May 2006

Accepted 8 August 2006
Ágnes Sonnevend, ${ }^{1,2}$ Vincent O. Rotimi, ${ }^{3}$ Jolanta Kolodziejek, ${ }^{4}$ Asad Usmani, ${ }^{1}$ Norbert Nowotny ${ }^{1,4}$ and Tibor Pál ${ }^{1,2}$

\author{
${ }^{1}$ Department of Medical Microbiology, Faculty of Medicine and Health Sciences, United Arab \\ Emirates University, Al Ain, United Arab Emirates \\ ${ }^{2}$ Department of Medical Microbiology and Immunology, University of Pécs, Pécs, Hungary \\ ${ }^{3}$ Department of Medical Microbiology, Faculty of Medicine, University of Kuwait, Kuwait \\ ${ }^{4}$ Zoonoses and Emerging Infections Group, Clinical Virology, Clinical Department of Diagnostic \\ Imaging, Infectious Diseases and Clinical Pathology, University of Veterinary Medicine, Vienna, \\ Austria
}

\begin{abstract}
The antibiotic sensitivity and the serotype and molecular type (MT) distribution of 41 Campylobacter jejuni strains isolated from individual patients in Tawam Hospital, Al Ain, United Arab Emirates, were investigated. While all strains were sensitive to erythromycin (MIC 0.5-4 mg I-1), 35 isolates (85.4\%) exhibited resistance to ciprofloxacin (MIC 8-64 $\mathrm{mg} \mathrm{l}^{-1}$ ). All resistant strains carried the Thr-86 to lle mutation in the gyrase $\mathrm{A}(\mathrm{gr} A \mathrm{~A})$ gene, as shown by mismatch amplification mutation assay (MAMA) and confirmed by sequencing. Based on the partial sequences of $g y r A$, resistant isolates carried 10 distinct alleles, eight of them representing new variants. Strains were assigned to $30 \mathrm{MTs}$ based on the combined results of PFGE and flaA PCR-RFLP typing. Eight of the 35 ciprofloxacin-resistant strains, isolated over a period of more than 1 year, represented the largest MT, also carrying the same allelic variant of the gyrA gene. These results show that the local incidence of fluoroquinolone resistance among $C$. jejuni is one of the highest reported worldwide. It was also demonstrated that stable MTs could persist for a relatively long time among the clonally unrelated antibiotic-resistant isolates of $C$. jejuni. The data also emphasize the need to replace fluoroquinolones as empirical therapy for diarrhoea of undiagnosed aetiology.
\end{abstract}

\section{INTRODUCTION}

Fluoroquinolone resistance in Campylobacter jejuni, a leading cause of gastrointestinal infections worldwide and one of the most important causes of travellers' diarrhoea, is rapidly increasing. Resistance is commonly due to point mutations in the quinolone resistance determinant region (QRDR) of the gyrase A (gyrA) gene involving the Thr-86 for high, and the Asp-90 and Ala-70 amino acid residues for lower-level resistance (Engberg et al., 2001).

While C. jejuni infections usually present as acute selflimiting gastrointestinal illnesses, in severe or prolonged

\footnotetext{
Abbreviations: MAMA, mismatch amplification mutation assay; MT, molecular type; QRDR, quinolone resistance determinant region; UAE, United Arab Emirates.

The GenBank/EMBL/DDBJ accession numbers for the partial sequences of the gyrA genes representing new allelic variants are DQ449657, DQ449658, DQ449659, DQ449660, DQ449661, DQ449662, DQ449663 and DQ449664.
}

cases antibiotic therapy is indicated, with macrolides as the primary drug of choice (Butzler, 2004). However, particularly if the causative agent is not identified, as is often the case in travellers' diarrhoea, fluoroquinolones are frequently used empirically (Guerrant et al., 2001; Yates, 2005). In such cases, resistance to ciprofloxacin can result in therapeutic failure (Guerrant et al., 2001; Sanders et al., 2002). Moreover, by mechanisms yet to be elucidated, fluoroquinolone resistance appears to coincide with increased fitness of the organism. In humans, fluoroquinolone-resistant strains are shed longer than sensitive isolates (Nelson et al., 2004; Engberg et al., 2004), and they have been reported to out-compete their sensitive counterparts, at least in certain genetic backgrounds, when colonizing poultry, i.e. the natural reservoir of the pathogen (Luo et al., 2005). All these findings underscore the necessity for close monitoring of the incidence and spread of fluoroquinolone-resistant clones of C. jejuni.

Countries on the Arabian Peninsula, some of them increasingly popular tourist destinations, have recently 
been scored as areas of high risk to acquire campylobacter infections (Ekdahl \& Andersson, 2004). However, data on the incidence of fluoroquinolone resistance and on the type of strains present in the region are very limited (Sjogren et al., 1989; Albert et al., 2005; Jumaa \& Neringer, 2005). The aim of the current study was to investigate the variety of $C$. jejuni strains isolated between 2002 and 2004 in a tertiary care hospital in Abu Dhabi Emirate, United Arab Emirates (UAE), and to determine the frequency and the genetic background of fluoroquinolone resistance among the local isolates.

\section{METHODS}

Strains and culture conditions. Forty-one C. jejuni strains isolated from faecal samples of individual patients in the Microbiology Laboratory of Tawam Hospital, Eastern Region of the Abu Dhabi Emirate, UAE, between September 2002 and September 2004 were studied. Tawam Hospital is a tertiary care teaching hospital, one of the two major hospitals in the city of $\mathrm{Al}$ Ain, which has a population of approximately 350000 . The 41 strains represented $63 \%$ of the 65 Campylobacter isolates recovered from the 3389 stool samples tested during this period of time. Seven of these strains were not available for us due to technical reasons, six represented repeated isolates and 11 were species other than $C$. jejuni. Four of the $41 C$. jejuni strains finally included in the study were recovered from inpatients. No detailed data (except the diagnosis 'diarrhoea' or 'gastroenteritis') on the medical conditions, therapeutic or travel history of the patients were available to us. Strains were stored in tryptic soy broth (Oxoid) containing $10 \%(\mathrm{v} / \mathrm{v})$ glycerol at $-80^{\circ} \mathrm{C}$, and were routinely cultured on campylobacter agar base (Oxoid) supplemented with $5 \%(\mathrm{v} / \mathrm{v})$ sheep blood, under microaerophilic conditions generated with CampyGen (Oxoid) bags in jars at $36{ }^{\circ} \mathrm{C}$ for $48 \mathrm{~h}$. The identification of the isolates was by colonial morphology and Gramstain characteristics, and confirmed by the API Campy kit (bioMérieux) according to the manufacturer's instructions.

Antibiotic susceptibility testing. The MICs of nalidixic acid, ciprofloxacin and erythromycin were determined by the agar dilution method using Mueller-Hinton agar (Oxoid) supplemented with $5 \%(\mathrm{v} / \mathrm{v})$ sheep blood and incubated under microaerophilic conditions at $36^{\circ} \mathrm{C}$ for $48 \mathrm{~h}$. The final antibiotic concentrations in the agar plates ranged from 0.25 to $512 \mathrm{mg} \mathrm{l}^{-1}$, according to the standards of the Clinical and Laboratory Standards Institute (CLSI; formerly known as the National Committee for Clinical Laboratory Standards, NCCLS) (NCCLS, 2003, 2005). Reference strain C. jejuni ATCC 33560 was included in each run as control.

Serotyping. Serotyping, based on the heat-stable antigen of the strains, was carried out by passive haemagglutination (Penner \& Hennessy, 1980) using a set of commercial antisera (Denka Seiken) according to the manufacturer's instructions.

DNA purification. Genomic DNA was purified with a QIAamp DNA Mini kit (Qiagen) according to the manufacturer's instructions.

Mismatch amplification mutation assay (MAMA) PCR and sequencing of the QRDR of gyrase A. The MAMA PCR detection of the Thr-86 to Ile mutation in the gyrA gene of the quinolone-resistant isolates was carried out according to the method of Zirnstein et al. (1999). A 673-bp-long part of the gyrA gene, containing the QRDR, was amplified using primers GZgyrA5 and GZgyrA6 (Zirnstein et al., 1999), and directly sequenced by fluorescence-based direct sequencing employing the ABI Prism Dye Terminator cycle sequencing ready reaction kit (Perkin-Elmer) and an ABI Prism 310 genetic analyser automated sequencing system (Perkin-Elmer). For the analysis of the sequences, the program MEGA version 3.1 (Kumar et al., 2004) was used.

PCR-RFLP. The amplification of the flaA gene using consensus primers, and the digestion of the amplicon with DdeI were carried out as described by Wassenaar \& Newell (2000). The digested products were run in a $2 \%$ agarose gel at $1 \mathrm{~V} \mathrm{~cm}^{-1}$ for $120 \mathrm{~min}$. Photographs of gels stained with ethidium bromide were analysed by the GelCompare II software (Applied Maths).

PFGE. C. jejuni genomic DNA was digested with SmaI following the formaldehyde inactivation of DNase, and the fragments were subsequently separated on a CHEF DRII apparatus (Bio-Rad) as described by the 'Campynet' PFGE Subtyping Subgroup (2000). The macrorestriction patterns of the isolates were compared according to the Dice similarity index (1-1\% tolerance interval) using the GelCompare II software. A PFGE group was arbitrarily defined as a strain if it exhibited macrorestriction band patterns with at least $95 \%$ similarity.

\section{RESULTS AND DISCUSSION}

\section{Antibiotic susceptibility and serotype distribution of the isolates}

All isolates were sensitive to erythromycin (MIC $\left.0 \cdot 5-4 \mathrm{mg} \mathrm{l}^{-1}\right)$, but 35 out of the 41 isolates $(85 \cdot 4 \%)$ were resistant to nalidixic acid and ciprofloxacin with MIC values of between 128 and $512 \mathrm{mg} \mathrm{l}^{-1}$, and 8 and $64 \mathrm{mg} \mathrm{l}^{-1}$, respectively.

Although fluoroquinolone resistance in campylobacter is rapidly emerging all over the world, high incidences comparable to the one found in our study have only been reported from a few locations, such as 88 and $75 \%$ in Spain (Ruiz et al., 1998; Saenz et al., 2000), $96 \%$ in Thailand (Sanders et al., 2002), 85.9\% in Hong Kong (Chu et al., 2004), and $77 \cdot 1 \%$ in India (Jain et al., 2005). Data from the Middle East are sparse. In 1998, Talhouk et al. (1998) reported a considerably lower level of ciprofloxacin resistance $(39 \%)$ in Lebanon among strains of human and animal origin. Recently, Albert et al. (2005) found $53 \%$ ciprofloxacin resistance among human campylobacter isolates in Kuwait. It should be noted that the results of both the Kuwaiti study and our study were based on strains collected at single hospital laboratories. Further, more extensive, larger-scale studies should reveal whether or not the current studies reflect real differences in the incidences of fluoroquinolone resistance in this pathogen between the northern, and south-eastern parts of the Arabian Peninsula.

Earlier, Jumaa \& Neringer (2005) had reported a $50 \%$ fluoroquinolone-resistance rate among the Campylobacter spp. isolated from humans between 1999 and 2002, from the same hospital laboratory. The use of different methodologies in their study and the current study prevents the direct comparison of the results. However, the possibility of a considerable rise in fluoroquinolone resistance in the 2 years that followed their report cannot be excluded, and should be proven by further monitoring of its incidence. 
A total of $31(75 \cdot 6 \%)$ of the 41 strains tested were serotypable, representing 10 serogroups. Serotype complex group HS4,13,16,43,50 was the most frequently encountered (22\%), followed by serotype HS2 (14.6\%); both are commonly encountered groups at other geographical locations also (Patton et al., 1991; Nielsen et al., 1997; Rautelin \& Hänninen, 1999; Wareing et al., 2002) (Fig. 1).

\section{Molecular typing of the isolates}

All strains were typable by both PFGE and PCR-RFLP. The 41 isolates belonged to 26 distinct PFGE groups (P1-P26); 21 of them consisting of single isolates only (Fig. 1). Two PFGE groups (P3 and P17) contained two, a further two types (P1 and P4) contained four, and a single type (P7) consisted of eight isolates. Of the 23 RFLP types (R1-R23), 15 were represented by single isolates only. Combining the results of the two typing methods, strains could be assigned to 30 distinct molecular types (MTs) (MT1-30). It is noteworthy that the eight isolates with identical PFGE patterns (P7) also exhibited identical RFLP patterns (R13) and formed a homogeneous MT (MT1) representing $19 \cdot 5 \%$ of the total, and $22 \cdot 8 \%$ of the ciprofloxacin-resistant strains (Fig. 1).

Similar to the results of others (Aarts et al., 1995; Rautelin \& Hänninen, 1999; Hänninen et al., 2001), we found that the overlap between the results of sero- and molecular typing was not complete. Identical serogroups were encountered in different P and R groups (e.g. HS2 in P4, P11, P12, P14 and P15, or in R6, R8, R10, R16 and R17), while different serogroups could be detected within the same molecular groups (HS2 and HS1,44 in P4, or HS10 and HS1,44 and HS4,13,16,43,50 in R7). On the other hand, six of the eight strains in the cluster MT1 belonged to serogroup HS4,13,16,43,50, while two isolates were non-typable (Fig. 1). The six strains sensitive to fluoroquinolones

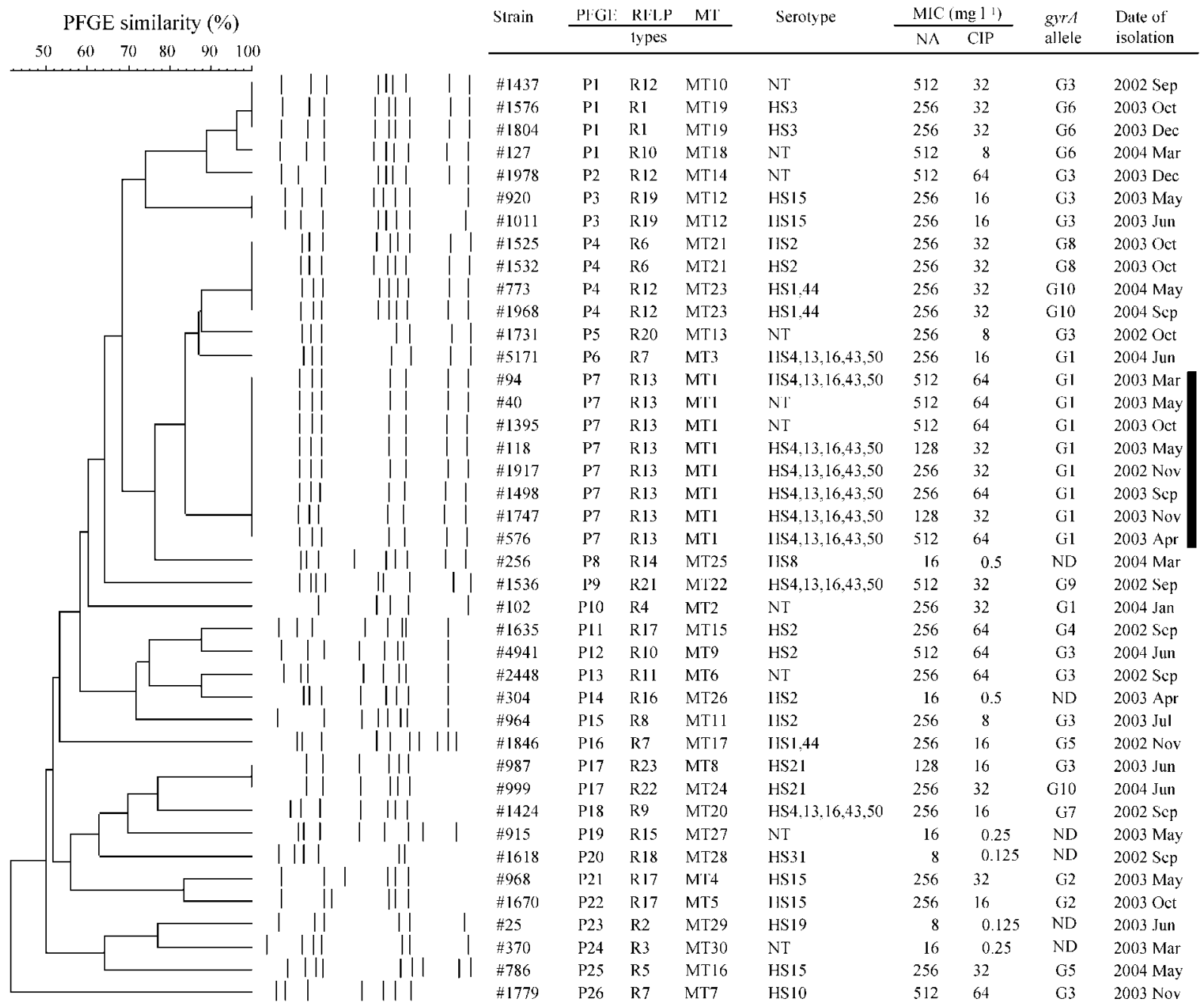

Fig. 1. MTs, sero- and allele types, antibiotic sensitivities and isolation dates of C. jejuni strains isolated in AI Ain, UAE. Abbreviations: NA, nalidixic acid; CIP, ciprofloxacin; NT, non-typable; ND, not done. Strains marked with the black bar represent members of a putative uniform clone. 
exhibited no clustering by either of the molecular typing methods or by serotyping (Fig. 1).

\section{Molecular basis of fluoroquinolone resistance}

All quinolone-resistant strains, but none of the sensitive ones, were positive by MAMA PCR, suggesting the presence of the Thr-86 to Ile mutation in the QRDR of their gyrA genes. The nucleotide sequence of the gyrA gene between nucleotides 64 and 654 (codons 22 and 218) confirmed the presence of the Thr-86 to Ile replacement in all fluoroquinolone-resistant isolates as the only amino acid change within the QRDR (i.e. between codons 69 and 120). These data, together with those recently published from Kuwait (Albert et al., 2005), show that, similar to other geographical locations (Zirnstein et al., 1999; Engberg et al., 2001; Hakanen et al., 2002; Dionisi et al., 2004; Beckmann et al., 2004), this mutation is the one most commonly encountered in fluoroquinolone-resistant isolates in the Middle East. Extensive polymorphism of the gyrA gene in C. jejuni within as well as outside the QRDR is well documented (Dionisi et al., 2004; Beckmann et al., 2004; Hakanen et al., 2002; Piddock et al., 2003). Based on partial sequences, the 35 fluoroquinolone-resistant strains encountered in this study carried 10 allelic variants of the gyrA gene (G1-G10), although distributed disproportionately (Fig. 1, Table 1). Allelic types G1 and G3 were carried by 10 isolates. A further two groups (G6 and G10) contained three strains, three groups (G2, G5 and G8) consisted of two strains, and three allelic variants (G4, G7 and G9) were represented by single isolates only. Surveying GenBank, only nucleotide sequences identical to allelic variants G3 and G10 were found (GenBank accession nos AJ567826.1 and AJ567825•1, respectively) (Table 1).

The clonal nature of strains in MT1, i.e. strains with identical PFGE and flaA-RFLP patterns, remains to be elucidated. The fact that all members of this MT also carried the same allelic variant of the highly polymorphic gyrA further corroborates the assumption that these strains, isolated over a year-long period of time (Fig. 1), are closely related. Clonal complexes can strongly associate with certain animal hosts, e.g. poultry (Manning et al., 2003), a commonly consumed food in the Middle East. These reservoirs, in turn, may serve as sources of human infection with strains exhibiting similar or identical geno- and phenotypes. On the other hand, stable $C$. jejuni clones isolated over an extended period of time from apparently independent sources have also been described (Manning et al., 2001, 2003). The lack of epidemiological data and information on the type distribution of local C. jejuni strains isolated from food and from potential animal reservoirs prevents us from clarifying the nature and epidemiological implications of this uniform cluster of strains within the framework of the current study. Nevertheless, their true clonal nature should be proven by sequence analysis of multiple loci of their genomes (Manning et al., 2003).

Table 1. Allelic variants of the gyrA gene of ciprofloxacin-resistant C. jejuni isolates

Amino acid abbreviations: S, serine; T, threonine; N, asparagine; A, alanine; G, glycine; I, isoleucine; V, valine.

\begin{tabular}{|c|c|c|c|c|c|c|c|c|c|c|c|c|c|c|c|c|c|c|c|c|c|c|c|c|}
\hline $\begin{array}{l}\text { gyrA } \\
\text { type }\end{array}$ & $\boldsymbol{n}^{\dagger}$ & $\begin{array}{c}\text { Nucleotide } \neq \\
\text { Codon } \S \\
\text { aa no.II }\end{array}$ & $\begin{array}{l}64 \\
\text { agt }\end{array}$ & $\begin{array}{l} \\
22 \\
(S)\end{array}$ & $\begin{array}{l}72 \\
\text { tat }\end{array}$ & $\begin{array}{l}84 \\
\text { tct }\end{array}$ & $\begin{array}{l}243 \\
\text { cac }\end{array}$ & $\begin{array}{l}257 \\
\text { aca }\end{array}$ & $\begin{array}{l}86 \\
(\mathrm{~T})\end{array}$ & $\begin{array}{l}330 \\
\text { ggc }\end{array}$ & $\begin{array}{l}357 \\
\text { agt }\end{array}$ & $\begin{array}{l}360 \\
\text { gcc }\end{array}$ & $\begin{array}{l}393408 \\
\text { agt gag }\end{array}$ & $\begin{array}{l}456 \\
\text { tat }\end{array}$ & $\begin{array}{l}4714 \\
\text { agc } \xi\end{array}$ & $\begin{array}{l}483558 \\
\text { gtt } \quad \text { cca }\end{array}$ & $\begin{array}{l}608 \\
\text { aat }\end{array}$ & 203 & $\begin{array}{l}616 \\
\text { gca }\end{array}$ & 206 & $\begin{array}{l}617 \\
\text { gca }\end{array}$ & 206 & $\begin{array}{l}622645 \\
\text { cta atc }\end{array}$ & $\begin{array}{c}\text { GenBank } \\
\text { accession } \\
\text { no. }\end{array}$ \\
\hline G1 & 10 & & & & c & & $\mathrm{t}$ & $\mathrm{t}$ & $\rightarrow \mathbf{I}$ & & c & $\mathrm{t}$ & & & $\mathrm{t}$ & c & g & $\rightarrow \mathrm{S}$ & $\mathrm{a}$ & $\rightarrow \mathbf{T}$ & & & $\mathrm{t}$ & DQ449657 \\
\hline G2 & 2 & & $\mathrm{~g}$ & $\rightarrow \mathbf{G}$ & $\mathrm{c}$ & & $\mathrm{t}$ & $\mathrm{t}$ & $\rightarrow \mathbf{I}$ & & c & $\mathrm{t}$ & & & $\mathrm{t}$ & c & g & $\rightarrow \mathrm{S}$ & $\mathrm{a}$ & $\rightarrow \mathrm{T}$ & & & $\mathrm{t}$ & DQ449658 \\
\hline G3 & 10 & & g & $\rightarrow \mathrm{G}$ & c & & $\mathrm{t}$ & $\mathrm{t}$ & $\rightarrow \mathbf{I}$ & & c & $\mathrm{t}$ & & & $\mathrm{t}$ & c & g & $\rightarrow \mathrm{S}$ & & & & & $\mathrm{t}$ & AJ567826•1 \\
\hline G4 & 1 & & $\mathrm{~g}$ & $\rightarrow \mathbf{G}$ & $\mathrm{c}$ & & $\mathrm{t}$ & $\mathrm{t}$ & $\rightarrow \mathrm{I}$ & & c & $\mathrm{t}$ & c & & $\mathrm{t}$ & c & g & $\rightarrow \mathrm{S}$ & & & & & $\mathrm{t}$ & DQ449659 \\
\hline G5 & 2 & & $\mathrm{~g}$ & $\rightarrow \mathbf{G}$ & $\mathrm{c}$ & & $\mathrm{t}$ & $\mathrm{t}$ & $\rightarrow \mathbf{I}$ & & c & $\mathrm{t}$ & & c & $\mathrm{t}$ & c & $\mathrm{g}$ & $\rightarrow \mathrm{S}$ & & & & & $\mathrm{t}$ & DQ449660 \\
\hline G6 & 3 & & & & c & $c$ & $\mathrm{t}$ & $\mathrm{t}$ & $\rightarrow \mathrm{I}$ & & c & $\mathrm{t}$ & & & $\mathrm{t}$ & c & $\mathrm{g}$ & $\rightarrow S$ & & & & & $\mathrm{t}$ & DQ449661 \\
\hline G7 & 1 & & $\mathrm{~g}$ & $\rightarrow \mathrm{G}$ & $\mathrm{c}$ & & $\mathrm{t}$ & $\mathrm{t}$ & $\rightarrow \mathrm{I}$ & $\mathrm{t}$ & c & $\mathrm{t}$ & & & $\mathrm{t}$ & c & g & $\rightarrow \mathrm{S}$ & & & $\mathrm{t}$ & $\rightarrow \mathrm{V}$ & $\mathrm{t}$ & DQ449662 \\
\hline G8 & 2 & & & & & & $\mathrm{t}$ & $\mathrm{t}$ & $\rightarrow \mathrm{I}$ & $\mathrm{t}$ & c & $\mathrm{t}$ & & & $\mathrm{t}$ & c & g & $\rightarrow \mathrm{S}$ & & & $\mathrm{t}$ & $\rightarrow \mathrm{V}$ & $\mathrm{t}$ & DQ449663 \\
\hline G9 & 1 & & & & & & & $\mathrm{t}$ & $\rightarrow \mathbf{I}$ & & c & & $\mathrm{a}$ & & $\mathrm{t}$ & g & & & & & & & $\mathrm{t}$ & DQ449664 \\
\hline G10 & 3 & & & & & & & $\mathrm{t}$ & $\rightarrow \mathrm{I}$ & & & & & & & & & & & & & & & AJ567825•1 \\
\hline
\end{tabular}

*Allelic variants of the partially sequenced gyrA gene.

$\dagger$ Number of isolates.

$\$$ Position of nucleotides numbered from the first nucleotide of the gyrA gene of C. jejuni NCTC 11168 (GenBank accession no. AL139077). $\S$ Codon with the affected base underlined.

IIThe position and symbol (in parentheses) of the amino acid replaced, numbered according to the deduced amino acid sequence of the gyrA gene of C. jejuni NCTC 11168 . 
Two further strains (\#5171 and \#102) carried the same allele of the gyrA gene as members of genotype group MT1, one of them belonged to serogroup complex HS4,13,16,43,50, while the other was non-typable (Fig. 1). However, these two isolates exhibited $<90 \%$ similarity by PFGE and $<70 \%$ similarity by PCR-RFLP to the other members of this group. The other large group of strains with an identical allele of the gyrA gene (G3) exhibited more extensive variations with the different typing techniques. They belonged to four different serogroups, and three isolates were non-typable. Furthermore, none of the isolates in this group exhibited similarities exceeding $85 \%$ by PFGE and $80 \%$ by PCR-RFLP to each other, as can be seen in Fig. 1 .

Our data show a high incidence $(85.4 \%)$ of fluoroquinolone resistance among $C$. jejuni strains isolated in Al Ain, UAE. This observation is not biased by the fact that eight, i.e. $22 \cdot 8 \%$, of the ciprofloxacin-resistant strains represented a single MT isolated over a considerable period of time. Even if members of MT1 had been counted as only one isolate, the incidence of fluoroquinolone resistance would still be $82.3 \%$, a very high figure by any comparison. This study, the first of this kind from the south-eastern part of the Arabian Peninsula, was too limited in scale to extrapolate the results to the whole UAE. Nevertheless, since some parts of the Middle East are increasingly frequented travel destinations, as well as relatively high-risk areas to acquire campylobacter infections (Ekdahl \& Andersson, 2004), the data should stress the need for the establishment of an effective antibiotic surveillance in the region. Furthermore, they also indicate that alternatives to fluoroquinolones for the treatment of fairly severe diarrhoeal cases with unconfirmed aetiology should be sought.

\section{ACKNOWLEDGEMENTS}

This work was supported by grants from UAE University/GCC (no. 018-965/03), from UAE University (no. 01-10-8-11/06) to T. P., and from Kuwait University (no. CM01/04) to V.O.R. We would like to express our appreciation to the personnel of the Microbiology Laboratory, Tawam Hospital, Al Ain, for providing the isolates.

\section{REFERENCES}

Aarts, H. J., Van Lith, L. A. \& Jacobs-Reitsma, W. F. (1995), Discrepancy between Penner serotyping and polymerase chain reaction fingerprinting of Campylobacter isolated from poultry and other animal sources. Lett Appl Microbiol 20, 371-374.

Albert, M. J., Neil, L., Pazhoor, A. A., Haridas, S., Rotimi, V. O. \& Khan, I. (2005). Ciprofloxacin resistance and its molecular mechanism in Campylobacter spp. isolated in Kuwait. Microb Drug Resist 11, 266-270.

Beckmann, L., Muller, M., Luber, P., Schrader, C., Bartelt, E. \& Klein, G. (2004). Analysis of gyrA mutations in quinolone-resistant and -susceptible Campylobacter jejuni isolates from retail poultry and human clinical isolates by non-radioactive single-strand conformation polymorphism analysis and DNA sequencing. J Appl Microbiol 96, 1040-1047.
Butzler, J. P. (2004). Campylobacter, from obscurity to celebrity. Clin Microbiol Infect 10, 868-876.

'Campynet' PFGE Subtyping Subgroup (2000). 'Campynet' Prototype Standardised Protocol for Pulse-Field Gel ElectrophoresisBased DNA Typing of Campylobacter jejuni and Campylobacter coli. http://campynet.vetinst.dk/PFGE.html.

Chu, Y. W., Chu, M. Y., Luey, K. Y., Ngan, Y. W., Tsang, K. L. \& Kam, K. M. (2004). Genetic relatedness and quinolone resistance of Campylobacter jejuni strains isolated in 2002 in Hong Kong. Clin Microbiol 42, 3321-3323.

Dionisi, A. M., Luzzi, I. \& Carattoli, A. (2004). Identification of ciprofloxacin-resistant Campylobacter jejuni and analysis of the gyrA gene by the LightCycler mutation assay. Mol Cell Probes 18, 255-261.

Ekdahl, K. \& Andersson, Y. (2004). Regional risks and seasonality in travel-associated campylobacteriosis. BMC Infect Dis 4, 54.

Engberg, J., Aarestrup, F. M., Taylor, D. E., Gerner-Smidt, P. \& Nachamkin, I. (2001). Quinolone and macrolide resistance in Campylobacter jejuni and C. coli: resistance mechanisms and trends in human isolates. Emerg Infect Dis 7, 24-34.

Engberg, J., Neimann, J., Nielsen, E. M., Aerestrup, F. M. \& Fussing, V. (2004). Quinolone-resistant Campylobacter infections: risk factors and clinical consequences. Emerg Infect Dis 10, 1056-1063.

Guerrant, R. L., Van Gilder, T., Steiner, T. S. \& 15 other authors (2001). Practice guidelines for the management of infectious diarrhea. Clin Infect Dis 32, 331-351.

Hakanen, A., Jalava, J., Kotilainen, P., Jousimies-Somer, H., Siitonen, A. \& Huovinen, P. (2002). gyrA polymorphism in Campylobacter jejuni: detection of gyrA mutations in 162 C. jejuni isolates by single-strand conformation polymorphism and DNA sequencing. Antimicrob Agents Chemother 46, 2644-2647.

Hänninen, M. L., Perko-Makela, P., Rautelin, H., Duim, B. \& Wagenaar, J. A. (2001). Genomic relatedness within five common Finnish Campylobacter jejuni pulsed-field gel electrophoresis genotypes studied by amplified fragment length polymorphism analysis, ribotyping, and serotyping. Appl Environ Microbiol 67, 1581-1586.

Jain, D., Sinha, S., Prasad, K. N. \& Pandey, C. M. (2005). Campylobacter species and drug resistance in a north Indian rural community. Trans $R$ Soc Trop Med Hyg 99, 207-214.

Jumaa, P. A. \& Neringer, R. (2005). A survey of antimicrobial resistance in a tertiary referral hospital in the United Arab Emirates. J Chemother 17, 376-379.

Kumar, S., Tamura, K. \& Nei, M. (2004). MEGA3: integrated software for molecular evolutionary genetics analysis and sequence alignment. Brief Bioinform 5, 150-163.

Luo, N., Pereira, S., Sahin, O., Lin, J., Huang, S., Michel, L. \& Zhang, Q. (2005). Enhanced in vivo fitness of fluoroquinolone-resistant Campylobacter jejuni in the absence of antibiotic selection pressure. Proc Natl Acad Sci U S A 102, 541-546.

Manning, G., Duim, B., Wassenaar, T., Wagenaar, J. A., Ridley, A. \& Newell, D. G. (2001). Evidence for a genetically stable strain of Campylobacter jejuni. Appl Environ Microbiol 67, 1185-1189.

Manning, G., Dowson, C. G., Bagnall, M. C., Ahmed, I. H., West, M. \& Newell, D. G. (2003). Multilocus sequence typing for comparison of veterinary and human isolates of Campylobacter jejuni. Appl Environ Microbiol 69, 6370-6379.

NCCLS (2003). Methods for Dilution Antimicrobial Susceptibility Tests for Bacteria that Grow Aerobically, approved standard, 6th edn. Document M7-A6. Wayne, PA: National Committee for Clinical Laboratory Standards.

NCCLS (2005). Performance Standards for Antimicrobial Susceptibility Testing, 15th information supplement. Document M100-S13. Wayne, PA: National Committee for Clinical Laboratory Standards. 
Nelson, J. M., Smith, K. E., Vugia, D. J. \& 8 other authors (2004). Prolonged diarrhea due to ciprofloxacin-resistant campylobacter infection. J Infect Dis 190, 1150-1157.

Nielsen, E. M., Engberg, J. \& Madsen, M. (1997). Distribution of serotypes of Campylobacter jejuni and C. coli from Danish patients, poultry, cattle and swine. FEMS Immunol Med Microbiol 19, 47-56.

Patton, C. M., Wachsmuth, I. K., Evins, G. M., Kiehlbauch, J. A., Plikaytis, B. D., Troup, N., Tompkins, L. \& Lior, H. (1991). Evaluation of 10 methods to distinguish epidemic-associated Campylobacter strains. J Clin Microbiol 29, 680-688.

Penner, J. L. \& Hennessy, J. N. (1980). Passive hemagglutination technique for serotyping Campylobacter fetus subsp. jejuni on the basis of soluble heat-stable antigens. J Clin Microbiol 12, 732-737.

Piddock, L. J., Ricci, V., Pumbwe, L., Everett, M. J. \& Griggs, D. J. (2003). Fluoroquinolone resistance in Campylobacter species from man and animals: detection of mutations in topoisomerase genes. J Antimicrob Chemother 51, 19-26.

Rautelin, H. \& Hänninen, M. L. (1999). Comparison of a commercial test for serotyping heat-stable antigens of Campylobacter jejuni with genotyping by pulsed-field gel electrophoresis. J Med Microbiol 48, 617-621.

Ruiz, J., Goni, P., Marco, F., Gallardo, F., Mirelis, B., Jimenez De Anta, T. \& Vila, J. (1998). Increased resistance to quinolones in Campylobacter jejuni: a genetic analysis of gyrA gene mutations in quinolone-resistant clinical isolates. Microbiol Immunol 42, 223-226.
Saenz, Y., Zarazaga, M., Lantero, M., Gastanares, M. J., Baquero, F. \& Torres, C. (2000). Antibiotic resistance in Campylobacter strains isolated from animals, foods, and humans in Spain in 1997-1998. Antimicrob Agents Chemother 44, 267-271.

Sanders, J. W., Isenbarger, D. W., Walz, S. E. \& 9 other authors (2002). An observational clinic-based study of diarrheal illness in deployed United States military personnel in Thailand: presentation and outcome of Campylobacter infection. Am J Trop Med Hyg 67, 533-538.

Sjogren, E., Johny, M. \& Kaijser, B. (1989). The serotype distribution of Campylobacter in patients with diarrhoea in Kuwait. FEMS Microbiol Lett 48, 237-239.

Talhouk, R. S., el-Dana, R. A., Araj, G. F., Barbour, E. \& Hashwa, F. (1998). Prevalence, antimicrobial susceptibility and molecular characterization of Campylobacter isolates recovered from humans and poultry in Lebanon. J Med Liban 46, 310-316.

Wareing, D. R., Bolton, F. J., Fox, A. J., Wright, P. A. \& Greenway, D. L. (2002). Phenotypic diversity of Campylobacter isolates from sporadic cases of human enteritis in the UK. J Appl Microbiol 92, 502-509.

Wassenaar, T. M. \& Newell, D. G. (2000). Genotyping of Campylobacter spp. Appl Environ Microbiol 66, 1-9.

Yates, J. (2005). Traveler's Diarrhea. Am Fam Physician 71, 2095-2100.

Zirnstein, G., Li, Y., Swaminathan, B. \& Angulo, F. (1999). Ciprofloxacin resistance in Campylobacter jejuni isolates: detection of gyrA resistance mutations by mismatch amplification mutation assay PCR and DNA sequence analysis. J Clin Microbiol 37, 3276-3328. 